\title{
BMJ Open Feasibility of wearable cameras to assess screen time and time spent restrained in children aged 3 to 5 years: a study protocol
}

\author{
Katherine L Downing, ${ }^{\oplus 1}$ Xanne Janssen, ${ }^{2}$ John $\mathrm{J}$ Reilly ${ }^{2}$
}

To cite: Downing KL, Janssen $\mathrm{X}$, Reilly JJ. Feasibility of wearable cameras to assess screen time and time spent restrained in children aged 3 to 5 years: a study protocol. BMJ Open 2019:9:e028265. doi:10.1136/ bmjopen-2018-028265

- Prepublication history and additional material for this paper are available online. To view these files, please visit the journal online (http://dx.doi. org/10.1136/bmjopen-2018028265).

Received 29 November 2018 Revised 29 April 2019 Accepted 29 April 2019

\section{Check for updates}

(c) Author(s) (or their employer(s)) 2019. Re-use permitted under CC BY-NC. No commercial re-use. See rights and permissions. Published by BMJ.

${ }^{1}$ Institute for Physical Activity and Nutrition, Deakin University, Burwood, Victoria, Australia

${ }^{2}$ School of Psychological Sciences and Health, University of Strathclyde, Glasgow, UK

Correspondence to Dr Katherine L Downing; k.downing@deakin.edu.au

\section{ABSTRACT}

Introduction Wearable cameras may help overcome the limitations of existing tools to measure young children's sedentary behaviour, but their use introduces a range of ethical challenges. The primary aim of this study is to determine the feasibility of using wearable cameras to measure the two aspects of sedentary behaviour currently included in evidence-based guidelines (ie, screen time and time spent restrained). If shown to be feasible, a secondary aim will be to validate subjective measures against the directly measured screen time and time spent restrained. Methods and analysis A convenience sample $(n=20)$ will be recruited via flyers at the University of Strathclyde and advertisements on online forums for parents of young children (aged 3 to 5 years). Children will be provided with a wearable camera, attached to the front of their clothing with a clip, to be worn for 3 days ( 2 non-childcare days and 1 weekend day) in non-public settings. Once switched on, the camera will record continuous video footage. Parents will complete an online survey providing their feedback on their own and their child's experience of the wearable camera. They will also report their own and their child's demographical characteristics and their child's usual daily screen time and time spent restrained in the past week. Data will be downloaded using specialised software and second-by-second coding will be undertaken. Feasibility and acceptability will be assessed using percentages and by analysing qualitative data. If feasibility is shown, intraclass coefficients will be used to determine agreement between video data and parent-reported sedentary behaviours.

Ethics and dissemination Ethical approval has been granted by the School of Psychological Sciences and Health Ethics Committee at the University of Strathclyde. Results will be used to inform future studies and will be disseminated in peer-reviewed journals and at major international conferences.

\section{INTRODUCTION}

For young children (under the age of 5 years), examples of sedentary behaviour include engaging in screen time (eg, television, computer, tablet, phone); reading, drawing, painting or other quiet play while sitting, reclining or lying and time spent

\section{Strengths and limitations of this study}

- This study will be the first to test the feasibility of using wearable cameras to objectively measure preschool children's screen time and time spent restrained.

- If feasibility is demonstrated and preliminary data on validity is obtained in the present study, findings will provide the basis of a larger and more definitive future validation study.

- Due to ethical constraints, cameras will not be worn in public settings so it is possible that any screen time or time spent restrained in these settings may not be captured.

restrained (eg, in car seats, high chairs or strollers). ${ }^{1} \quad$ Parent-reported screen time is the most commonly studied sedentary behaviour in this age group and has been consistently associated with increased risk of overweight/obesity, poor psychosocial health and decreased cognitive development. ${ }^{2}{ }^{3}$ There is emerging evidence that, in addition to minimising opportunities to be active and reducing opportunities for more useful sedentary behaviours (involving interactions with family members), time spent restrained may be adversely associated with overweight/ obesity. ${ }^{4}$ It is these two detrimental sedentary behaviours that are the focus of the current study.

Given the associated adverse health outcomes, public health guidelines recommend limiting time spent being sedentary. In 2017, Canada and Australia co-released the first 24 hours movement guidelines for young children. ${ }^{56}$ The guidelines recommend that preschool children ( 3 to 5 years) should accumulate at least 180 min of physical activity (of which at least $60 \mathrm{~min}$ is energetical play), sleep for 10 to 13 hours, not be restrained (eg, in a stroller or car seat) for more than $60 \mathrm{~min}$ at a time and have no more than $60 \mathrm{~min}$ of screen 
time per day. Similar guidelines are under development in South Africa, the UK and at the WHO. ${ }^{7}$

In order to not only determine compliance with these guidelines, but also examine the health outcomes associated with these movement behaviours and to evaluate the effectiveness of interventions, valid and reliable measurement tools are required. ${ }^{8}$ Accelerometers are commonly used to provide an objective measure of usual physical activity and sleep and have been validated in children. ${ }^{9-11}$ Although accelerometers can also be used to objectively assess total sedentary time (as a lack of movement) or total sitting, there are currently no objective measures of the specific sedentary behaviours included in public health guidelines (ie, screen time and time spent restrained). Compliance and public health surveillance therefore depends largely on subjective parent-report. Currently none of the subjective measures of measurement of sedentary behaviour have been validated against objective measures, in part because of technical barriers and in part because of ethical considerations (ie, objective measures of these behaviours rely on direct observation or filming). Parent-report may also be subject to social desirability and recall bias. ${ }^{12}{ }^{13}$ Additionally, with the pervasive nature of screens (eg, smart phones and tablets),${ }^{14}$ it may be challenging for parents to accurately estimate the sum of their child's screen time in a single global assessment. It also may not be possible for parents to report on these behaviours when they are not with their children (eg, in childcare settings or with other carers).

Wearable cameras are increasingly being used in health behaviour research. Although still relatively new, they have been successfully used with adults to objectively measure physical activity and sedentary behaviour ${ }^{15-17}$ and behavioural nutrition. ${ }^{18} 19$ The use of wearable cameras in health behaviour research introduces a host of ethical issues, primarily relating to the confidentiality and security of the data collected, the intrusive nature of the devices, the likelihood that unflattering or unwanted data may be captured and that both participants and third parties may feel uncomfortable having their image recorded. ${ }^{20}$ These issues may be even more problematical in studies with children and families than in studies with adults. However, very recently, at least some wearable cameras have been shown to be feasible for use with school-aged and adolescent children to study dietary intake, ${ }^{21}$ exposure to food ${ }^{22} 23$ and alcohol ${ }^{24}$ marketing, the world in which children live, ${ }^{25}$ residential neighbourhoods and other destinations ${ }^{26}$ and travel to school. ${ }^{27}$ They have also been used to examine the early obesogenic home environment (worn by parents of 2- to 8-year-old children) ${ }^{28}$ and with infants and parents to measure infants' exposure to faces ${ }^{29}$ and parent-infant interactions. ${ }^{30}$ To our knowledge, no studies have utilised wearable cameras in preschool children.

Despite the novelty of wearable cameras and the associated ethical considerations, in all of the recent studies wearable cameras were shown to be feasible and acceptable. Wearable cameras provide a cost-effective and less intrusive alternative to researcher direct observation. They may help overcome the limitations of existing tools used to measure preschool children's screen time and time spent restrained; however, there are numerous ethical challenges associated with collecting this type of data in this population. Although previous studies utilising wearable cameras have received ethics approval, they include little discussion of the steps taken to obtain approval and any challenges encountered. This paper describes the study protocol, including discussion of the ethical issues we encountered. The primary aim of the study is to determine the feasibility of using wearable cameras to measure the two aspects of sedentary behaviour currently included in evidence-based guidelines, that is, preschool children's screen time and time spent restrained. If shown to be feasible, a secondary aim will be to validate subjective measures against the directly measured screen time and time spent restrained.

\section{METHODS AND ANALYSIS}

\section{Patient and public involvement}

This study protocol was designed without patient involvement; patients were not invited to comment on recruitment or the study design. However, given that this is a feasibility study, patient input will be incorporated into future larger studies.

\section{Recruitment}

Participants will be recruited in Glasgow, Scotland, through convenience sampling and online advertisements. Flyers will be posted on noticeboards at the University of Strathclyde and advertisements posted on online forums for parents of young children (eg, https:// www.mumsnet.com). Recruitment and data collection will take place between September 2019 and June 2020.

\section{Inclusion criteria}

Inclusion criteria will be: having a child aged 3 to 5 years, able to freely give informed consent and English-language competency.

\section{Sample size}

As a pilot/feasibility study, this study will aim to recruit 20 parent-child dyads.

\section{Measures}

\section{Choice of camera}

Previous studies have used a range of different wearable cameras. Microsoft SenseCam (https://www.microsoft. $\mathrm{com} / \mathrm{en}-\mathrm{us} / \mathrm{research} / \mathrm{project} / \mathrm{sensecam} /$ ), the most commonly used of these, was developed specifically for research purposes and is not available commercially. We attempted to contact Microsoft about the possibility of borrowing SenseCam cameras but were not able make contact. The Vicon Revue and OMG Autographer were developed based on the SenseCam and were produced for several years under license; however, they are no longer available. For the present study, we investigated the other 
commercially available wearable cameras and considered size, battery life, encryption capability, availability and cost. Given the target population of preschoolers, the size and weight of the camera were particularly important for comfort and safety. The battery life also needed to be sufficient to allow for continuous recording for up to 12 hours per day. It was also a requirement for ethics approval to ensure the camera and data were secure (ie, encrypted so that only the researchers could access the data). This is discussed more in the 'Ethical considerations' section below. Finally, the cost of the camera was an important consideration given that no external funding was received for this study. The PR6 wearable camera (PR6 body worn camera, Pinnacle Response; https://www.pinnacleresponse.com/pr6-body-worn-camera) appears to be the only available camera which met all of these criteria and hence will be utilised in the current study. It is small (99 $\mathrm{mm} \times 58 \mathrm{~mm} \times 23 \mathrm{~mm}$ ), lightweight (155 grams), has 13.5 hour recording capability, includes AES256 encryption, has a water and dust rating of IP66 (ie, water resistant against powerful jets) and is drop resistant from 3 metres. The PR6 takes continuous video footage, which differs to most previously used cameras that capture still images every $30 \mathrm{~s}$. However, continuous footage has the potential to provide a more accurate measure of children's behaviours given that second-by-second coding can be undertaken.

\section{Data collection}

Children and their parents will be visited either at their home or at the University of Strathclyde, where children will be provided with a wearable camera. The camera will be attached to the front of the child's clothing with a clip, with a safety-release lanyard around the neck for additional security (eg, if the camera is to fall off). Parents will be asked to ensure that their child wears the camera for a total of 3 days: 2 non-childcare days (ie, days that the child is at home with the parent) and 1 weekend day. Given the 13.5 hour recording capacity of the camera (described above), it is possible that the cameras may not have the capacity to record three full days of data. This will be dependent on the daily wear time and will be an aspect of feasibility that is considered. A study examining the feasibility of wearable cameras to examine the early obesogenic home environment (with participants asked to wear the camera during waking hours while at home for four consecutive days) reported a mean wear time of 5.9 (SD 2.6) hours. ${ }^{28}$ Based on this, we expect that wear times in our sample will be similar. Children will be asked to wear the camera only in the home environment or other settings where members of the public are unlikely to be photographed (eg, in the car, at friends' homes). The wear period was chosen as it is considered to be sufficient to capture usual behaviour while minimising participant burden. Parents will also be provided with a charger and asked to charge the camera overnight or on non-wear days. Once switched on, the camera will record continuous video footage (without audio).
At the end of the 3 days parents will be asked to complete an online survey (comprising quantitative and qualitative questions) providing their feedback on their own and their child's experience of the wearable camera, based on previous literature examining wearable camera feasibility. ${ }^{28}$ They will be asked to report on the ease of use, awareness of the camera, reactions from others (ie, family and friends), any instances where they or their child chose not to wear the camera and the appropriateness of the amount of time the child wore the camera. Parents will also report demographics (child and parent date of birth, sex, height and weight) and their child's usual daily screen time and time spent restrained in the past week while at home. The screen time and time spent restrained items are from a larger international study (currently in development).

\section{Ethical considerations}

Kelly et al published an ethical framework for automated, wearable cameras in health behaviour research, ${ }^{20}$ to which this study adheres. The framework includes a checklist of guidelines for research using wearable cameras in free-living observational studies. The way in which these guidelines were or will be addressed in the present study, and any additional ethical considerations from the School of Psychological Sciences and Health Ethics Committee (SEC), are outlined below. A copy of the checklist is provided in online supplementary table 1.

\section{Informed written consent of participants}

The participant information sheet provided at the time of collecting written informed consent will contain all of information detailed in the guidelines. This includes: (1) the number of hours of video footage recorded, (2) the nature and type of data that can be collected with an automated, wearable camera, (3) that participants may forget that they are wearing the device and record unwanted and unflattering footage (eg, bathroom visits), (4) that data of illegal activities may not be protected by confidentiality, (5) that no individual will be identifiable in any research dissemination, (6) that participants will have the opportunity to view (and delete if necessary) the recorded footage in privacy prior to the research team viewing the images, (7) that participants are able to remove the device whenever they wish, (8) that participants will not get copies of their images and (9) that only a team of specifically trained researchers will have access to the image data.

\section{Privacy and confidentiality}

The camera that will be utilised in the present study was chosen based on a number of criteria as described above, the most important of which was the security of the images recorded. This was raised as an important issue for the SEC, in case of loss of the camera. Many commercially available wearable cameras do not offer encryption of images, which means that if the camera is lost it would be possible for anyone to access and view the images. The PR6 camera that will be used in this study 
has secure encryption and password-protection, so that only the research team will be able to access the video data. Neither participants nor third parties will be able to access the footage. For additional security in case the camera falls off the child's clothing, the camera will be attached to a safety-release lanyard (designed to break away from the neck if caught on anything, to minimise any risk of choking/strangulation).

All video footage will be stored securely in a password-protected folder, on the University of Strathclyde's secure cloud-type storage application according to the UK Data Protection Act. The ethical framework states that the camera should be configured to allow participants to cease recording for short periods. Although this is not possible with the camera that we will use, participants will be informed that they can turn the device around, or simply switch it off and on again, in any situation. Both parents and children will be informed that they should remove the device in settings where it may not be permitted or appropriate to record, or when privacy is required. They will be also given the opportunity to note specific times when they want footage removed and to review and delete any footage (using the software provided with the cameras) under the guidance of the research team. Finally, only the investigators on the study will have access to the video data to ensure participant confidentiality.

\section{Non-maleficence}

When applying for ethical approval for the present study, a major concern of the SEC was that video footage of members of the public would be recorded. Existing guidelines state that it is not practical or necessary to obtain informed consent when taking images of individuals and groups in public spaces, unless the images are published or disseminated in such a way that they can be recognised. ${ }^{31}{ }^{32}$ However, it was decided that initially, to test the feasibility of the cameras to measure preschool children's sedentary behaviour, children would only wear the camera in the home environment or other non-public settings (eg, in the car, at friends' homes). If shown to be feasible, future studies would have the camera worn during all waking hours, which would provide a more comprehensive measure of the behaviours of interest (eg, children may have screen time or be restrained in public settings such as restaurants). Parents and children will be provided with the following short explanation, in the event that they forget to remove the camera in a public setting, 'My child is/I am volunteering for a research project looking at measuring sedentary behaviour. The device records my child's/my daily activities. We/I can remove it if you are not comfortable'.

\section{Autonomy of third parties}

Participants will be asked to seek verbal permission from all family members and cohabitants, given the likelihood that their image will be recorded. They will also be provided with advice for informing friends and acquaintances about the device and its purpose, and will be told that they can remove it if anyone is uncomfortable with their image being recorded. Participants will be informed that they can offer for any third party (friends, acquaintances, etc) to have footage deleted. They will either be able to record the dates/times of any footage third parties want deleted, or can provide third parties with contact details for the research team. Finally, no video footage captured for this study will be published.

\section{Data management}

When the camera is returned, video data will be downloaded using specialised software that is provided with the PR6 camera. Data will be coded using video coding software, which will generate a time stamp every time the presence of screen time (TV viewing, computer use, electronic game use, tablet computer use or smartphone use) or time spent restrained (in a car seat, stroller/ pram or high chair) is manually coded. Every second following a given time stamp will be coded as being the same activity as that occurring at the point of the time stamp itself. Each second will be coded in this way until a change in activity is indicated, resulting in second-bysecond coding. Coding will be completed by three coders (KLD, XJ, JJR). Intra-rater reliability will be assessed by coders recoding a randomly selected day of footage they originally coded, and inter-rater reliability will be assessed by coders recoding a randomly selected day of footage originally coded by another coder. The total amount of screen time on individual days will be determined and a weighted average will be calculated to determine average daily screen time. The same process will be followed for time spent restrained.

\section{Statistical analyses}

All analyses will be conducted using Stata 15 (StataCorp, College Station, Texas, USA). Descriptive statistics will be used to describe the sample. Feasibility and acceptability will be assessed using percentages and by analysing qualitative data, as appropriate. Depending on the length of responses given to qualitative survey items by parents, NVivo 12 (QSR International) qualitative software package may be used to conduct thematic analyses. The use of the cameras will be deemed feasible if parent questionnaire data suggests that the burden on them/their child was acceptable $(\geq 70 \%$ of the sample responding positively to the survey items), and 3 days of recording (for at least 1 hour per day) is achieved in at least $75 \%(15 / 20)$ of the sample. Participant retention will also be determined as an indicator of feasibility. If feasibility is shown, preliminary evidence on validation will be determined. Intraclass coefficients (ICCs) will be used to determine agreement between video data and parent-reported screen time and time spent restrained behaviours. ICCs will be defined as: $<0.40=$ poor, 0.40 to $0.59=$ fair, 0.60 to $0.74=\operatorname{good}$ and 0.75 to $1.00=$ excellent. $^{33}$ 


\section{DISCUSSION}

The present study will be the first to determine the feasibility of wearable cameras to measure young children's screen time and time spent restrained. To date, surveillance data have relied on parent-report of children's time in these behaviours, which can be difficult to recall and may be subject to social desirability and recall bias. ${ }^{12} 13$ With the release of new 24 hours movement guidelines for the early years that suggest a healthy balance of sleep, physical activity and sedentary behaviour (ie, screen time and time spent restrained), it is vitally important to have valid and reliable quantitative measures of these specific behaviours. If child screen time and time spent restrained cannot be quantified by simple parent report, or if parent report can only provide relative measures of these important sedentary behaviour variables (eg, rank-order correlations with more accurate objective measures) then it is important that this is known.

With the exception of two studies conducted $>10$ years ago (one validating television diaries against video observations $^{34}$ and one validating parental questionnaires against an automated TV monitoring device $\mathrm{e}^{35}$ ), no studies have validated parent-report of sedentary behaviours. If feasibility is demonstrated and preliminary data on validity is obtained in the present study, findings will provide the basis of a larger and more definitive future validation study. If validity from this and future studies is high, surveillance data can continue to rely on parent-report. The feasibility results from this study (from the perspective of both the participants and the researchers) may also have wider use in wearable camera research. Additionally, the ethical challenges faced in the development of this protocol are not frequently reported and may be useful for future studies as research with wearable cameras increases.

There are some limitations of the current study. First, due to ethical constraints, cameras will not be worn in public settings. It is therefore possible that some screen time or time spent restrained may not be measured, for example, if children are using screens such as iPads in restaurants. However, if feasibility is found to be acceptable in this study we plan to conduct future studies with cameras worn during all waking hours. Additionally, because the cameras will only be worn in the home environment and participants' daily time spent in the home will vary (eg, some participants may be home for only 2 hours per day whereas others may be home for the entire day), it is challenging to set a daily minimum wear time criterion. As such, a daily minimum wear time criterion of 1 hour was decided to maximise the inclusion of data (but to exclude those with minimal data that may not be representative of a usual day). A possible limitation is that the large amount of data that will be recorded may result in errors during coding. We will assess both intra- and interrater reliability to minimise any source of error. Finally, being a feasibility study the findings may not be generalisable. Selection bias may be introduced given that we plan to recruit a convenience sample. Additionally, there is the possibility of the Hawthorne effect, whereby participants will know the purpose of the study and may modify their own (and their child's) behaviour in response. This may have implications for the validity aspect of the current study. Replication of the study in a larger sample and in different populations, such as low socioeconomical areas and in different cultures and contexts (eg, low- and middle-income countries), will be necessary. It may also be important for future, larger studies to consider participant blinding to mitigate the possibility of the Hawthorne effect.

\section{Ethics and dissemination}

The protocol adheres to Kelly et al ethical framework for automated, wearable cameras in health behaviour research ${ }^{20}$; see 'Ethical considerations' section above. Parents will provide written, informed consent and children will provide verbal assent to take part in the study. Parents will also be asked to obtain verbal permission from all family members and cohabitants, since they will likely be photographed. The findings will be published in a peer-reviewed journal article and will be used to inform larger studies (if found to be feasible).

Contributors XJ and JJR conceived the idea for the study; XJ, JJR and KLD designed the study. KLD drafted the manuscript; XJ and JJR critically revised the article. All authors read and approved the final manuscript.

Funding KLD is supported by an Australian Government Department of Education and Training Endeavour Research Fellowship.

Competing interests None declared.

Patient consent for publication Not required.

Ethics approval The study has been granted ethical approval through the School of Psychological Sciences and Health Ethics Committee at the University of Strathclyde (project number UEC18/65).

Provenance and peer review Not commissioned; externally peer reviewed.

Open access This is an open access article distributed in accordance with the Creative Commons Attribution Non Commercial (CC BY-NC 4.0) license, which permits others to distribute, remix, adapt, build upon this work non-commercially, and license their derivative works on different terms, provided the original work is properly cited, appropriate credit is given, any changes made indicated, and the use is non-commercial. See: http://creativecommons.org/licenses/by-nc/4.0/.

\section{REFERENCES}

1. Tremblay MS, Aubert S, Barnes JD, et al. Sedentary Behavior Research Network (SBRN) - Terminology Consensus Project process and outcome. Int J Behav Nutr Phys Act 2017;14:75.

2. Poitras VJ, Gray CE, Janssen X, et al. Systematic review of the relationships between sedentary behaviour and health indicators in the early years (0-4 years). BMC Public Health 2017;17:868.

3. Carson V, Kuzik N, Hunter S, et al. Systematic review of sedentary behavior and cognitive development in early childhood. Prev Med 2015;78:115-22.

4. Sijtsma A, Sauer PJ, Stolk RP, et al. Infant movement opportunities are related to early growth--GECKO Drenthe cohort. Early Hum Dev 2013;89:457-61.

5. Tremblay MS, Chaput JP, Adamo KB, et al. Canadian 24-Hour Movement Guidelines for the Early Years (0-4 years): An Integration of Physical Activity, Sedentary Behaviour, and Sleep. BMC Public Health 2017;17(Suppl 5):874.

6. Okely AD, Ghersi D, Hesketh KD, et al. A collaborative approach to adopting/adapting guidelines - The Australian 24-Hour Movement Guidelines for the early years (Birth to 5 years): an integration of physical activity, sedentary behavior, and sleep. BMC Public Health 2017;17(Suppl 5):869. 
7. Okely AD, Tremblay MS, Reilly JJ, et al. Physical activity, sedentary behaviour, and sleep: movement behaviours in early life. Lancet Child Adolesc Health 2018;2:233-5.

8. Colley RC, Garriguet D, Adamo KB, et al. Physical activity and sedentary behavior during the early years in Canada: a crosssectional study. Int J Behav Nutr Phys Act 2013;10:54.

9. Janssen X, Cliff DP, Reilly JJ, et al. Predictive validity and classification accuracy of ActiGraph energy expenditure equations and cut-points in young children. PLoS One 2013;8:e79124.

10. Kinder JR, Lee KA, Thompson $\mathrm{H}$, et al. Validation of a hip-worn accelerometer in measuring sleep time in children. $J$ Pediatr Nurs 2012;27:127-33.

11. Galland B, Meredith-Jones K, Gray A, et al. Criteria for nap identification in infants and young children using 24-h actigraphy and agreement with parental diary. Sleep Med 2016;19:85-92.

12. Lubans DR, Hesketh K, Cliff DP, et al. A systematic review of the validity and reliability of sedentary behaviour measures used with children and adolescents. Obes Rev 2011;12:781-99.

13. Reilly JJ, Penpraze V, Hislop J, et al. Objective measurement of physical activity and sedentary behaviour: review with new data. Arch Dis Child 2008;93:614-9.

14. Pew Research Center. Mobile Fact Sheet. Washington, DC 2018.

15. Doherty AR, Kelly P, Kerr J, et al. Use of wearable cameras to assess population physical activity behaviours: an observational study. The Lancet 2012;380:S35.

16. Kerr J, Marshall SJ, Godbole S, et al. Using the SenseCam to improve classifications of sedentary behavior in free-living settings. Am J Prev Med 2013;44:290-6.

17. Oliver M, Doherty AR, Kelly $\mathrm{P}$, et al. Utility of passive photography to objectively audit built environment features of active transport journeys: an observational study. Int J Health Geogr 2013;12:20.

18. Gemming L, Doherty A, Utter J, et al. The use of a wearable camera to capture and categorise the environmental and social context of self-identified eating episodes. Appetite 2015;92:118-25.

19. Gemming L, Rush E, Maddison R, et al. Wearable cameras can reduce dietary under-reporting: doubly labelled water validation of a camera-assisted 24 h recall. Br J Nutr 2015;113:284-91.

20. Kelly P, Marshall SJ, Badland H, et al. An ethical framework for automated, wearable cameras in health behavior research. Am J Prev Med 2013;44:314-9.

21. Beltran A, Dadabhoy H, Ryan C, et al. Dietary Assessment with a Wearable Camera among Children: Feasibility and Intercoder Reliability. J Acad Nutr Diet 2018;118:2144-53.

22. Barr M, Signal L, Jenkin G, et al. Capturing exposures: using automated cameras to document environmental determinants of obesity. Health Promot Int 2015;30:56-63.
23. Signal LN, Stanley J, Smith M, et al. Children's everyday exposure to food marketing: an objective analysis using wearable cameras. Int J Behav Nutr Phys Act 2017;14:137.

24. Chambers T, Pearson AL, Stanley J, et al. Children's exposure to alcohol marketing within supermarkets: An objective analysis using GPS technology and wearable cameras. Health Place 2017;46:274-80.

25. Signal LN, Smith MB, Barr M, et al. Kids'Cam: An Objective Methodology to Study the World in Which Children Live. Am J Prev Med 2017;53:e89-e95.

26. Chambers T, Pearson AL, Kawachi I, et al. Kids in space: Measuring children's residential neighborhoods and other destinations using activity space GPS and wearable camera data. Soc Sci Med 2017;193:41-50

27. Kelly P, Doherty AR, Hamilton A, et al. Evaluating the feasibility of measuring travel to school using a wearable camera. Am J Prev Med 2012;43:546-50.

28. Schrempft S, van Jaarsveld $\mathrm{CH}$, Fisher A. Exploring the Potential of a Wearable Camera to Examine the Early Obesogenic Home Environment: Comparison of SenseCam Images to the Home Environment Interview. J Med Internet Res 2017;19:e332.

29. Sugden NA, Mohamed-Ali MI, Moulson MC. I spy with my little eye: typical, daily exposure to faces documented from a first-person infant perspective. Dev Psychobiol 2014;56:249-61.

30. Lee R, Skinner A, Bornstein MH, et al. Through babies' eyes: Practical and theoretical considerations of using wearable technology to measure parent-infant behaviour from the mothers and infants' view points. Infant Behav Dev 2017;47:62-71.

31. Wiles R, Prosser J, Bagnoli A, et al. Visual ethics: ethical issues in visual research. National Centre for Research Methods: Southampton, 2008.

32. British Sociological Association. Statement of ethical practice for the British Sociological Association - Visual Sociology Group. Durham 2006.

33. Guidelines CDV. criteria, and rules of thumb for evaluating normed and standardized assessment instruments in psychology. Psychological Assessment 1994;6:284-90.

34. Anderson DR, Field DE, Collins PA, et al. Estimates of young children's time with television: a methodological comparison of parent reports with time-lapse video home observation. Child Dev 1985;56:1345-57.

35. Robinson JL, Winiewicz DD, Fuerch JH, et al. Relationship between parental estimate and an objective measure of child television watching. Int J Behav Nutr Phys Act 2006;3:43. 